\title{
The effect of an external toxicant on a biological species in case of deformity: a model
}

\author{
Anuj Kumar Agarwal ${ }^{1}$ A. W. Khan ${ }^{1}$ A. K. Agrawal $^{2}$
}

Received: 16 July 2016/ Accepted: 25 July 2016/Published online: 5 August 2016

(C) Springer International Publishing Switzerland 2016

\begin{abstract}
In this paper, a mathematical model is proposed and analyzed to study the effect of an external toxicant on a biological species. Here, we have considered that the toxicant is constantly emitted in the environment form some external source and after-effect of this external toxicant some members of biological species shows deformity as incapable in reproduction. The analytical results of model system are established by stability analysis and Hopf-bifurcation theory. The model's results show, when emission of external toxicant increases, total population density decreases and density of deformed subclass increases. For highly emission of external toxicant, system become unstable and shows a supercritical Hopf-bifurcation. To verify the analytical results, a numerical simulation is provided.
\end{abstract}

Keywords Mathematical model - Biological species · Toxicant $\cdot$ Deformity $\cdot$ Hopf-bifurcation

Mathematics Subject Classification 34C60 - 37L10 . 92D25 $\cdot 93 \mathrm{~A} 30$

Anuj Kumar Agarwal

anuj.kumaragarwal20@gmail.com

A. W. Khan

awkhan@iul.ac.in

A. K. Agrawal

akagrawal@lko.amity.edu

1 Department of Mathematics, Integral University, Lucknow, Uttar Pradesh, India

2 Department of Mathematics, Amity University, Lucknow, Uttar Pradesh, India

\section{Introduction}

Mathematical models are used in large-scale to predict the various nature of real life problems; e.g. in ecology, epidemiology, ecotoxicology and other many problems. Many researchers also used mathematical models to predict the growth of biological species in toxic environment. They have proposed and analyzed mathematical models by considering different cases, such as effect of a single toxicant or more than one toxicant on biological species, allelopathy case, deformity in a subclass of species, etc. (Freedman and Shukla 1991; Shukla and Agrawal 1999; Shukla et al. 2003; Agrawal and Shukla 2012; Kumar et al. 2016), to provide important insights for the effect of toxicants on biological species. In particular, Freedman and Shukla (1991) have studied the effect a single toxicant on a species with a consideration that toxicant affected on the growth rate and decreasing the carrying capacity of the environment. Shukla and Agrawal (1999) have proposed a model by considering a situation in which toxicant emitted by a biological species and decreased the density of other biological species (case of allelopathy). As an interesting observable fact, Agrawal and Shukla (2012) studied a model for the after-effect of a single toxicant a subclass of biological species shows deformity as incapable in reproduction. Here, it is assumed that toxicant is emitted in the environment from some external source. Further, understand the case of deformity after-effect of a toxicant in more meaningful manner, Kumar et al. (2016) proposed and analyzed a model with an assumption that toxicant emitted by biological species itself. They have shown, toxicant decreased the total population density and a subclass of species suffers from deformity. For higher emission rate, the model system becomes unstable. 
In this paper, we proposed and analyzed a mathematical model to study the effect of an external toxicant on a biological species a subclass of which is severely affected and gets deformed. This case is similar to the case studied by Agrawal and Shukla (2012). But, the proposed model in this study is a modified version of the model by Agrawal and Shukla (2012). The results obtained by modified model are more closer to real life in case of deformity. In this study, we also check the existence of hopf-bifurcation and the nature of bifurcating periodic solutions.

\section{Mathematical model}

We assume a biological species of population density $N(t)$ at time $t$, is logistically growing and surviving in a polluted environment. This polluted environment having a toxicant which is constantly emitted in the environment from some external sources. The environmental concentration of this toxicant is $T(t)$ at time $t$. We assume that $U(t)$ is the concentration of toxicant $T(t)$, taken up by the biological species $N(t)$ at time $t$. This toxicant is decreasing the growth rate of species $N(t)$ as well as a subclass of species with population density $N_{D}(t)$ shows deformity as incapability in reproduction. The remaining population density which is free from deformity is assumed as $N_{A}(t)$. Keeping these facts in mind, we propose the following mathematical model:

$$
\begin{aligned}
\frac{d N_{A}}{d t}= & (b-d) N_{A}-r_{1} U N_{A}-\frac{r N_{A} N}{K(T)} \\
\frac{d N_{D}}{d t}= & r_{1} U N_{A}-\frac{r N_{D} N}{K(T)}-(\alpha+d) N_{D} \\
\frac{d T}{d t}= & Q-\delta T-\gamma T N+\pi v N U \\
\frac{d U}{d t}= & \gamma T N-\beta U-v N U \\
N(t)= & N_{A}(t)+N_{D}(t), \quad N_{A}(0), N_{D}(0), T(0) \geq 0, \\
& U(0) \geq c T(0), \quad c>0, \quad 0 \leq \pi \leq 1
\end{aligned}
$$

All the parameters considered in the model are positive constants. $b$ and $d$ are the natural birth and death rate of biological species. $r$ represents the intrinsic growth rate of biological species. $Q$ is the rate at which external toxicant is constantly emitted in the environment. The external toxicant $T(t)$ is uptaken by the species at the rate $\gamma$. Aftereffect of this toxicant, the deformed-free population density decreases at the rate $r_{1} . \alpha$ is the mortality rate of deformed population due to high toxicity in the environment. $\delta$ and $\beta$ are naturally depletion rates of $T(t)$ and $U(t)$ respectively. $U(t)$ is depleted at the rate $v$ due to die out of some members of species and a fraction $\pi$ of this depletion is reentered into the environment. $c>0$ is a proportionality constant used to calculate the initial uptake concentration of toxicant. $K(T)$ is a decreasing function of $T$ to measure the carrying capacity of the environment.

i.e.

$K(0)=K_{0}>0, \quad K(T)>0, \quad \frac{d K}{d T}<0, \quad$ for $\mathrm{T}>0$

where $K_{0}$ is the carrying capacity of the toxic free environment.

To make the model system (1) free from $N_{A}(t)$, we reduce it into following model system (3) by using the fact that $N(t)=N_{A}(t)+N_{D}(t)$.

$$
\begin{aligned}
\frac{d N}{d t} & =r N-\frac{r N^{2}}{K(T)}-(\alpha+b) N_{D} \\
\frac{d N_{D}}{d t} & =r_{1} U\left(N-N_{D}\right)-\frac{r N_{D} N}{K(T)}-(\alpha+d) N_{D} \\
\frac{d T}{d t} & =Q-\delta T-\gamma T N+\pi v N U \\
\frac{d U}{d t} & =\gamma T N-\beta U-v N U
\end{aligned}
$$

The region of attraction $\Omega$ that attracts all the positive solution of model system (3), is as follows:

$$
\begin{aligned}
\Omega= & \left\{\left(N, N_{D}, T, U\right): 0 \leq N \leq K_{0}, 0 \leq N_{D} \leq \frac{r_{1} Q K_{0}}{\left(r_{1} Q+\delta_{m}(\alpha+d)\right)},\right. \\
& \left.0 \leq T+U \leq \frac{Q}{\delta_{m}}\right\}
\end{aligned}
$$

where $\delta_{m}=\min (\delta, \beta)$.

The proof of region of attraction $\Omega$ is given in Appendix A.

\section{Equilibrium points}

The model system (3) have two non-negative equilibrium points $E_{1}\left(0,0, \frac{Q}{\delta}, 0\right)$ and $E_{2}\left(N^{*}, N_{D}^{*}, T^{*}, U^{*}\right)$. Here, the existence of $E_{1}$ is understandable, hence omitted. The existence of $E_{2}$ is as follows:

The values $N^{*}, N_{D}^{*}, T^{*}$ and $U^{*}$ of the equilibrium point $E_{2}$ are the positive solutions of the following non-linear equations,

$$
\begin{aligned}
& N=\frac{\left(r-r_{1} U\right) K(T)}{r} \\
& N_{D}=\frac{r_{1} U N K(T)}{r N+\left(r_{1} U+\alpha+d\right) K(T)} \\
& T=\frac{Q(\beta+v N)}{f(N)}=g(N)(\text { say }) \\
& U=\frac{Q \gamma N}{f(N)}=h(N)(\text { say) }
\end{aligned}
$$


where

$$
f(N)=\delta \beta+(\gamma \beta+\delta v) N+\gamma v(1-\pi) N^{2}
$$

The Eq. (4c) shows that $T$ is directly proportional to the parameter $Q$ and from the Eq. (2), carrying capacity $K(T)$ decreases as $T$ increase. Hence, the carrying capacity of the environment decreases when the emission rate of external toxicant $Q$ increases.

Let

$F(N)=r N-\left(r-r_{1} h(N)\right) K(g(N))$

at $N=0$,

$F(0)=-r K\left(\frac{Q}{\delta}\right)<0$

at $N=K_{0}$

$F\left(K_{0}\right)=r K_{0}-\left(r-r_{1} h\left(K_{0}\right)\right) K\left(g\left(K_{0}\right)\right)>0$

Eqs. (6) and (7) show that $F(N)=0$ has a solution in the interval $\left[0, K_{0}\right]$.

Also, The root $N^{*}$ of $F(N)=0$ is unique, if

$\frac{d F}{d N}=\left[r+r_{1} K(g(N)) \frac{d h}{d N}-\left(r-r_{1} h(N)\right) \frac{d K}{d T} \frac{d g}{d N}\right]>0$

from, Eqs. (4c) and (4d)

$\frac{d g}{d N}=-\frac{Q \gamma}{f^{2}(N)}\left\{\beta^{2}+2 \beta v(1-\pi) N+v^{2}(1-\pi) N^{2}\right\}<0$

$\frac{d h}{d N}=\frac{Q \gamma}{f^{2}(N)}\left\{\delta \beta-\gamma \nu(1-\pi) N^{2}\right\}$

since, $\frac{d K}{d T}<0$ (from (2)) and $\frac{d g}{d N}$ (from (9a))

so,

$\left[\left(r-r_{1} h(N)\right)\left\{\frac{d K}{d T} \frac{d g}{d N}\right\}\right]>0$

The equation $F(N)=0$ has a unique root $N^{*}$, only when

$$
\left[r+r_{1} K(g(N)) \frac{d h}{d N}\right]>\left[\left(r-r_{1} h(N)\right) \frac{d K}{d T} \frac{d g}{d N}\right]
$$

Hence, $F(N)=0$ has a unique root $N^{*}$ in the interval $\left[0, K_{0}\right]$, if condition (10) hold. The values $N_{D}^{*}, T^{*}$ and $U^{*}$ can be find out using the value of $N^{*}$ in the Eq. (4).

\section{Dynamical behavior}

This section shows the dynamical behavior of model system (3) corresponding to the equilibrium points $E_{1}$ and $E_{2}$.

\section{Dynamical behavior corresponding to $E_{1}$}

The Jacobian matrix $M_{1}$ corresponding to the equilibrium points $E_{1}$ is as follows

$$
M_{1}=\left[\begin{array}{cccc}
r & -(\alpha+b) & 0 & 0 \\
0 & -(\alpha+d) & 0 & 0 \\
-\frac{\gamma Q}{\delta} & 0 & -\delta & 0 \\
\frac{\gamma Q}{\delta} & 0 & 0 & -\beta
\end{array}\right]
$$

Since, one of the eigenvalue of Jacobian matrix $M_{1}$ is $r>0$ and all the other eigenvalues are $-(\alpha+d),-\delta,-\beta<0$, which confirm that $E_{1}$ is a saddle point locally unstable manifold in $N$ - direction and locally stable manifold in $N_{D}-T-U$ space.

\section{Dynamical behavior corresponding to $E_{2}$}

To study the dynamical behavior, we linearize the model system (3) corresponding to the equilibrium point $E_{2}=$ $\left(N^{*}, N_{D}^{*}, T^{*}, U^{*}\right)$ by taking the following transformation:

$N=N^{*}+n, \quad N_{D}=N_{D}^{*}+n_{d}, \quad T=T^{*}+\tau, \quad U=U^{*}+u$.

here, $n, n_{d}, \tau$ and $u$ are taken as small perturbations around $E_{2}$.

So, the model system (3) can be written in the terms of $n, n_{d}, \tau$ and $u$ as follows:

$\dot{X}=M_{2} X+N_{2}$

where,

$X=\left[\begin{array}{c}n \\ n_{d} \\ \tau \\ u\end{array}\right], \quad M_{2}=\left[\begin{array}{cccc}m_{11} & m_{12} & m_{13} & 0 \\ m_{21} & m_{22} & m_{23} & m_{24} \\ m_{31} & 0 & m_{33} & m_{34} \\ m_{41} & 0 & m_{43} & m_{44}\end{array}\right], \quad N_{2}=\left[\begin{array}{c}n_{1} \\ n_{2} \\ n_{3} \\ n_{4}\end{array}\right]$

Moreover,

$m_{11}=-r\left\{\frac{2 N^{*}}{K\left(T^{*}\right)}-1\right\}, \quad m_{12}=-(\alpha+b), \quad m_{13}=\frac{r N^{* 2} K^{\prime}\left(T^{*}\right)}{K^{2}\left(T^{*}\right)}$,

$m_{21}=r_{1} U^{*}-\frac{r N_{D}^{*}}{K\left(T^{*}\right)}, \quad m_{22}=-\frac{r_{1} N^{*} U^{*}}{N_{D}^{*}}, \quad m_{23}=\frac{r N^{*} N_{D}^{*} K^{\prime}\left(T^{*}\right)}{K^{2}\left(T^{*}\right)}$,

$m_{24}=r_{1}\left(N^{*}-N_{D}^{*}\right), \quad m_{31}=\left[-\gamma T^{*}+\pi v U^{*}\right], \quad m_{33}=-\frac{\left(Q+\pi v N^{*} U^{*}\right)}{T^{*}}$,

$m_{34}=\pi v N^{*}, \quad m_{41}=\gamma T^{*}-v U^{*}, \quad m_{43}=\gamma N^{*}, m_{44}=-\left(\beta+v N^{*}\right)$,

$n_{1}=-\frac{r}{K\left(T^{*}\right)} \cdot n^{2}+\frac{r K^{\prime}\left(T^{*}\right)}{K^{2}\left(T^{*}\right)} \cdot n^{2} \tau+2 r N^{*} \frac{K^{\prime}\left(T^{*}\right)}{K^{2}\left(T^{*}\right)} \cdot n \tau$

$n_{2}=r_{1} \cdot u\left(n-n_{d}\right)-\frac{r}{K\left(T^{*}\right)} \cdot n n_{d}+\frac{r K^{\prime}\left(T^{*}\right)}{K^{2}\left(T^{*}\right)}\left[N_{D}^{*} \cdot n+N^{*} \cdot n_{d}+n n_{d}\right] \cdot \tau$

$n_{3}=-\gamma . n \tau+\pi v . n u, n_{4}=\gamma . n \tau-v . n u$.

In the Eq. (11), $M_{2} X$ and $N_{2}$ are showing the linear and non-linear parts of the model system (3) respectively and 
$M_{2}$ is a Jacobian matrix corresponding to the equilibrium point $E_{2}$. Thus, the characteristic equation of $M_{2}$ can be written as:

$p(x)=x^{4}+c_{1} x^{3}+c_{2} x^{2}+c_{3} x+c_{4}$

where,

$$
\begin{aligned}
c_{1}= & -\left(m_{11}+m_{22}+m_{33}+m_{44}\right) \\
c_{2}= & m_{11}\left(m_{22}+m_{33}+m_{44}\right)+m_{22}\left(m_{33}+m_{44}\right) \\
& +m_{33} m_{44}-\left(m_{34} m_{43}+m_{12} m_{21}+m_{13} m_{31}\right) \\
c_{3}= & \left(m_{33}+m_{44}\right)\left(m_{12} m_{21}-m_{11} m_{22}\right)+\left(m_{11}+m_{22}\right) \\
& \left(m_{34} m_{43}-m_{33} m_{44}\right)-m_{12}\left(m_{23} m_{31}+m_{24} m_{41}\right) \\
& +m_{13} m_{31}\left(m_{22}+m_{44}\right)-m_{13} m_{34} m_{41} \\
c_{4}= & \left(m_{11} m_{22}-m_{12} m_{21}\right)\left(m_{33} m_{44}-m_{34} m_{43}\right) \\
& +m_{12} m_{24}\left(m_{41} m_{33}-m_{31} m_{43}\right) \\
& +\left(m_{31} m_{44}-m_{34} m_{41}\right)\left(m_{12} m_{23}-m_{13} m_{22}\right)
\end{aligned}
$$

Applying the Routh-Hurwitz Criterion on the characteristic Eq. (12), all the eigenvalues of Jacobian matrix $M_{2}$ are either negative or having negative real parts iff

$c_{j}>0, \quad(j=1, \ldots, 4)$

$H_{2}=c_{1} c_{2}-c_{3}>0$

$H_{3}=c_{1} c_{2} c_{3}-c_{3}^{2}-c_{1}^{2} c_{4}>0$

Hence, we can state the following theorem to set up the local asymptotically stablility corresponding to the equilibrium point $E_{2}$.

Theorem 1 The equilibrium point $E_{2}$ of model system (3) is locally asymptotically stable under the conditions (13).

\section{Existence of Hopf-bifurcation}

The model system (3), has a possibility of Hopf-bifurcation (Hassard et al. 1981; Kuznetsov 2004; Seydel 2009) corresponding to the equilibrium point $E_{2}$. By treating $Q$ (i.e. the emission rate of external toxicant) as a bifurcation parameter, we check the existence of Hopf-bifurcation. It is obvious that a Hopf-bifurcation may exist if all the eigenvalues of Jacobian matrix are having negative real parts except a purely imaginary complex conjugate pair. In this case, the Jacobian matrix $M_{2}$ having four eigenvalues $x_{j}=R_{j}+i I_{j}(j=1, \ldots, 4)$ (say). So, the Hopf-bifurcation exist only when $R_{1}, R_{2}=0, I_{1}=$ $-I_{2} \neq 0 \& R_{3}, R_{4}<0$ at the critical value $Q=Q^{*}$ (say).

According to the Liu's criterion (Liu 1994), the model system (3) undergoes a Hopf-bifurcation at the critical value $Q=Q^{*}>0$, if

$$
\begin{aligned}
& {\left[c_{j}\right]_{Q=Q^{*}}>0 \text { for }(\mathrm{j}=1, \ldots, 4)} \\
& {\left[H_{2}\right]_{Q=Q^{*}}=\left[c_{1} c_{2}-c_{3}\right]_{Q=Q^{*}}>0} \\
& {\left[H_{3}\right]_{Q=Q^{*}}=\left[c_{1} c_{2} c_{3}-c_{3}^{2}-c_{1}^{2} c_{4}\right]_{Q=Q^{*}}=0} \\
& {\left[\frac{d R_{j}}{d Q}\right]_{Q=Q^{*}} \neq 0, \quad \text { for } \mathrm{j}=1,2}
\end{aligned}
$$

A New Detecting Method For Conditions of Existence of Hopf-bifurcation (Jiaqi and Zhujun 1995) describe the last condition (14d) in the terms of coefficients of characteristic Eq. (12) as follows:

$$
\left[\frac{d R}{d Q}\right]_{Q=Q^{*}}=\left[\frac{\frac{d}{d Q}\left(c_{1} c_{2} c_{3}-c_{3}^{2}-c_{1}^{2} c_{4}\right)}{2 c_{1}\left(4 c_{4}-c_{1} c_{3}-c_{2}^{2}\right)}\right]_{Q=Q^{*}} \neq 0
$$

Hence, the following theorem establishes the existence of Hopf-bifurcation corresponding to the bifurcation parameter $Q$.

Theorem 2 The model (3) undergoes a Hopf-bifurcation corresponding to the equilibrium point $E_{2}$, if $Q$ crosses the critical value $Q^{*}>0$ such that

1. $\left[c_{j}\right]_{Q=Q^{*}}>0$ for $(\mathrm{j}=1, \ldots, 4)$

2. $\left[H_{2}\right]_{Q=Q^{*}}=\left[c_{1} c_{2}-c_{3}\right]_{Q=Q^{*}}>0$

3. $\left[H_{3}\right]_{Q=Q^{*}}=\left[c_{1} c_{2} c_{3}-c_{3}^{2}-c_{1}^{2} c_{4}\right]_{Q=Q^{*}}=0$

4. $\left[\frac{d R}{d Q}\right]_{Q=Q^{*}}=\left[\frac{\frac{d}{d Q}\left(c_{1} c_{2} c_{3}-c_{3}^{2}-c_{1}^{2} c_{4}\right)}{2 c_{1}\left(4 c_{4}-c_{1} c_{3}-c_{2}^{2}\right)}\right]_{Q=Q^{*}} \neq 0$

Since, there exist a Hopf-bifurcation at the critical value of $Q=Q^{*}$. So, the model system (3) having bifurcating periodic solutions. In the next section, we examine the nature of bifurcating periodic solutions.

\section{Nature of bifurcating periodic solutions}

The direction and stability of bifurcating periodic solutions are examined by observing the sign of coefficients $\mu_{2}, \beta_{2}, \tau_{2}$ at the critical value of $Q=Q^{*}$, given by Hassard et al. (1981). We derived some explicit formulae for these coefficients using normal form and center manifold theory (Hassard et al. 1981; Kuznetsov 2004). At the critical value $Q=Q^{*}$, the eigenvalues of Jacobian matrix $M_{2}$ can be assumed as $\pm i v,-J_{1}$ and $-J_{2}$.

Let $X=P Y$, then the normal form is

$$
\dot{Y}=J Y+F, \quad Y=\operatorname{col} .\left(y_{1}, y_{2}, y_{3}, y_{4}\right)
$$

where 
$J=P^{-1} M_{2} P=\left[\begin{array}{cccc}0 & -v & 0 & 0 \\ v & 0 & 0 & 0 \\ 0 & 0 & -J_{1} & 0 \\ 0 & 0 & 0 & -J_{2}\end{array}\right] \quad$ and

$F=P^{-1} f=\left[\begin{array}{l}F_{1}\left(y_{1}, y_{2}, y_{3}, y_{4}\right) \\ F_{2}\left(y_{1}, y_{2}, y_{3}, y_{4}\right) \\ F_{3}\left(y_{1}, y_{2}, y_{3}, y_{4}\right) \\ F_{4}\left(y_{1}, y_{2}, y_{3}, y_{4}\right)\end{array}\right]$

Here,

$$
n=\sum_{j=1}^{4} P_{1 j} y_{j}, \quad n_{d}=\sum_{j=1}^{4} P_{2 j} y_{j}, \quad \tau=\sum_{j=1}^{4} P_{3 j} y_{j}, \quad u=\sum_{j=1}^{4} P_{4 j} y_{j}
$$

Now, we evaluated the following quantities at critical value of parameter $Q=Q^{*}$ and $\left(y_{1}, y_{2}, y_{3}, y_{4}\right)=(0,0,0,0)$.

Here $P$ is a transformed matrix defined as:

$$
P=\left[\begin{array}{llll}
P_{11} & P_{12} & P_{13} & P_{14} \\
P_{21} & P_{22} & P_{23} & P_{24} \\
P_{31} & P_{32} & P_{33} & P_{34} \\
P_{41} & P_{42} & P_{43} & P_{44}
\end{array}\right]
$$

$$
\begin{aligned}
g_{11}= & \frac{1}{4}\left\{\left(\frac{\partial^{2} F_{1}}{\partial y_{1}^{2}}+\frac{\partial^{2} F_{1}}{\partial y_{2}^{2}}\right)+i\left(\frac{\partial^{2} F_{2}}{\partial y_{1}^{2}}+\frac{\partial^{2} F_{2}}{\partial y_{2}^{2}}\right)\right\}, \\
g_{02}= & \frac{1}{4}\left\{\left(\frac{\partial^{2} F_{1}}{\partial y_{1}^{2}}-\frac{\partial^{2} F_{1}}{\partial y_{2}^{2}}-2 \frac{\partial^{2} F_{2}}{\partial y_{1} \partial y_{2}}\right)\right. \\
& \left.+i\left(\frac{\partial^{2} F_{2}}{\partial y_{1}^{2}}-\frac{\partial^{2} F_{2}}{\partial y_{2}^{2}}+2 \frac{\partial^{2} F_{1}}{\partial y_{1} \partial y_{2}}\right)\right\}, \\
g_{20}= & \frac{1}{4}\left\{\left(\frac{\partial^{2} F_{1}}{\partial y_{1}^{2}}-\frac{\partial^{2} F_{1}}{\partial y_{2}^{2}}+2 \frac{\partial^{2} F_{2}}{\partial y_{1} \partial y_{2}}\right)\right. \\
& \left.+i\left(\frac{\partial^{2} F_{2}}{\partial y_{1}^{2}}-\frac{\partial^{2} F_{2}}{\partial y_{2}^{2}}-2 \frac{\partial^{2} F_{1}}{\partial y_{1} \partial y_{2}}\right)\right\}, \\
g_{21}= & G_{21}+\sum_{j=1}^{2}\left(2 G_{110}^{j} w_{11}^{j}+G_{101}^{j} w_{20}^{j}\right)
\end{aligned}
$$

$P_{11}=m_{12} L_{1}, \quad P_{12}=m_{12} L_{2}, \quad P_{13}=m_{12} K_{31}, \quad P_{14}=m_{12} K_{32}$,

$P_{21}=-\left(m_{11} L_{1}+m_{13} L_{4}+v L_{2}\right), \quad P_{22}=\left(v L_{1}+v m_{13} m_{31}-m_{11} L_{2}\right)$,

$P_{23}=-\left[\left(m_{11}+J_{1}\right) K_{31}+m_{13} K_{11}\right], \quad P_{24}=-\left[\left(m_{11}+J_{2}\right) K_{32}+m_{13} K_{12}\right]$,

$P_{31}=m_{12} L_{4}, \quad P_{32}=-v m_{12} m_{31}, \quad P_{33}=m_{12} K_{11}, \quad P_{34}=m_{12} K_{12}$

$P_{41}=m_{12} L_{3}, \quad P_{42}=-v m_{12} m_{41}, \quad P_{43}=m_{12} K_{21}, \quad P_{44}=m_{12} K_{22}$

Also,

$L_{1}=m_{34} m_{43}-m_{33} m_{44}+v^{2}, \quad L_{2}=v\left(m_{33}+m_{44}\right)$,

$L_{3}=m_{33} m_{41}-m_{31} m_{43}, \quad L_{4}=m_{31} m_{44}-m_{34} m_{41}$

For $j=1,2$

$$
\begin{aligned}
K_{1 j}= & m_{31} m_{44}-m_{34} m_{41}+m_{31} J_{j}, \\
& K_{2 j}=m_{33} m_{41}-m_{31} m_{43}+m_{41} J_{j}, \\
K_{3 j}= & \left(m_{34} m_{43}-m_{33} m_{44}\right)-\left(m_{33}+m_{44}\right) J_{j}-J_{j}^{2} .
\end{aligned}
$$

and

$$
f=\left[\begin{array}{l}
f_{1}\left(y_{1}, y_{2}, y_{3}, y_{4}\right) \\
f_{2}\left(y_{1}, y_{2}, y_{3}, y_{4}\right) \\
f_{3}\left(y_{1}, y_{2}, y_{3}, y_{4}\right) \\
f_{4}\left(y_{1}, y_{2}, y_{3}, y_{4}\right)
\end{array}\right]
$$

where,

$$
\begin{aligned}
f_{1}\left(y_{1}, y_{2}, y_{3}, y_{4}\right)= & -\frac{r}{K\left(T^{*}\right)} \cdot n^{2}+\frac{r K^{\prime}\left(T^{*}\right)}{K^{2}\left(T^{*}\right)} \cdot n^{2} \tau+2 r N^{*} \frac{K^{\prime}\left(T^{*}\right)}{K^{2}\left(T^{*}\right)} \cdot n \tau \\
f_{2}\left(y_{1}, y_{2}, y_{3}, y_{4}\right)= & r_{1} \cdot u\left(n-n_{d}\right)-\frac{r}{K\left(T^{*}\right)} \cdot n n_{d} \\
& +\frac{r K^{\prime}\left(T^{*}\right)}{K^{2}\left(T^{*}\right)}\left[N_{D}^{*} \cdot n+N^{*} \cdot n_{d}+n n_{d}\right] \cdot \tau
\end{aligned}
$$

$f_{3}\left(y_{1}, y_{2}, y_{3}, y_{4}\right)=-\gamma \cdot n \tau+\pi v . n u$

$f_{4}\left(y_{1}, y_{2}, y_{3}, y_{4}\right)=\gamma . n \tau-v . n u$

where,

$$
\begin{aligned}
G_{21}= & \frac{1}{8}\left\{\left(\frac{\partial^{3} F_{1}}{\partial y_{1}^{3}}+\frac{\partial^{3} F_{2}}{\partial y_{2}^{3}}+\frac{\partial^{3} F_{2}}{\partial y_{1}^{2} \partial y_{2}}+\frac{\partial^{3} F_{1}}{\partial y_{1} \partial y_{2}^{2}}\right)\right. \\
& \left.+i\left(\frac{\partial^{3} F_{2}}{\partial y_{1}^{3}}-\frac{\partial^{3} F_{1}}{\partial y_{2}^{3}}-\frac{\partial^{3} F_{1}}{\partial y_{1}^{2} \partial y_{2}}+\frac{\partial^{3} F_{2}}{\partial y_{1} \partial y_{2}^{2}}\right)\right\}
\end{aligned}
$$

For $j=1,2$

$$
\begin{aligned}
G_{110}^{j}= & \frac{1}{2}\left\{\left(\frac{\partial^{2} F_{1}}{\partial y_{1} \partial y_{(j+2)}}+\frac{\partial^{2} F_{2}}{\partial y_{2} \partial y_{(j+2)}}\right)\right. \\
& \left.+i\left(\frac{\partial^{2} F_{2}}{\partial y_{1} \partial y_{(j+2)}}-\frac{\partial^{2} F_{1}}{\partial y_{2} \partial y_{(j+2)}}\right)\right\} \\
G_{101}^{j}= & \frac{1}{2}\left\{\left(\frac{\partial^{2} F_{1}}{\partial y_{1} \partial y_{(j+2)}}-\frac{\partial^{2} F_{2}}{\partial y_{2} \partial y_{(j+2)}}\right)\right. \\
& \left.+i\left(\frac{\partial^{2} F_{2}}{\partial y_{1} \partial y_{(j+2)}}+\frac{\partial^{2} F_{1}}{\partial y_{2} \partial y_{(j+2)}}\right)\right\} \\
h_{11}^{j}= & \frac{1}{4}\left(\frac{\partial^{2} F_{(j+2)}}{\partial y_{1}^{2}}+\frac{\partial^{2} F_{(j+2)}}{\partial y_{2}^{2}}\right), \\
h_{20}^{j}= & \frac{1}{4}\left(\frac{\partial^{2} F_{(j+2)}}{\partial y_{1}^{2}}-\frac{\partial^{2} F_{(j+2)}}{\partial y_{2}^{2}}-2 i \frac{\partial^{2} F_{(j+2)}}{\partial y_{1} \partial y_{2}}\right), \\
w_{11}^{k}= & \frac{h_{11}^{j}}{J_{k}}, \quad w_{20}^{j}=\frac{h_{20}^{j}}{J_{j}+2 i v}
\end{aligned}
$$

Now, we evaluate the coefficients $\mu_{2}, \beta_{2}$ and $\tau_{2}$ using the following formulae: 
$C_{1}(0)=\frac{i}{2 v}\left[g_{20} g_{11}-2\left|g_{11}\right|^{2}-\frac{1}{3}\left|g_{02}\right|^{2}\right]+\frac{1}{2} g_{21}$,

$\mu_{2}=-\frac{\operatorname{Re} C_{1} 0}{\operatorname{Re} x_{1}^{\prime}}$,

$\beta_{2}=2 \operatorname{Re} C_{1}(0), \quad \tau_{2}=-\frac{\left(\operatorname{Im~} C_{1}(0)+\mu_{2} \operatorname{Im} x_{1}^{\prime}\right)}{v}$,

Hence, the following theorem express the nature of bifurcating periodic solutions.

Theorem 3 If $\mu_{2}>0$ (or $\left.\mu_{2}<0\right)$, the model system (3) shows a supercritical (or subcritical) Hopf-bifurcation and the bifurcating periodic solutions exist for $Q>Q^{*}$ (or $Q<Q^{*}$ ), if $\beta_{2}<0$ (or $\beta_{2}>0$ ), the bifurcating periodic solutions are stable (or unstable), if $\tau_{2}>0$ (or $\left.\tau_{2}<0\right)$, the period of bifurcating solutions increases (or decreases).

\section{Numerical simulation}

We provide numerical simulation to back up our analytical results for the model system (3). A matlab package MATCONT (Dhooge et al. 2003) is used for the graphical representation of model system (3).

We assume, the carrying capacity function as

$K(T)=K_{0}-\frac{b_{1} T}{1+b_{2} T}$

and a set of parameters as:

$b=0.55, \quad d=0.0006, \quad r_{1}=0.8, \quad Q=0.006$,

$\alpha=0.0002, \quad \delta=0.08, \quad \gamma=0.005$,

$\pi=0.02, \quad v=0.0002, \quad \beta=0.08, \quad K_{0}=10.0$,

$b_{1}=0.02, \quad b_{2}=1.0$
The equilibrium point $E_{2}$ contains the value

$N^{*}=9.5989, N_{D}^{*}=0.3834, T^{*}=0.04689, U^{*}=0.02747$.

The condition (10) holds and $\frac{d F}{d N}=0.5495>0$, which show that $N^{*}$ is unique, in addition $E_{2}$ is unique. The local stability conditions (13) corresponding to $E_{2}$ are also satisfied.

Figure 1 shows the total density and density of deformed subclass of biological species corresponding to the parameter $Q$ (the remaining parameters are same as (19b)). The Fig. 1 shows that when the emission rate of external toxicant increases, the total density $N$ decrease and the density of deformed subclass $N_{D}$ firstly increase then decrease with $N$. For large emission rate $Q(=0.950)$ both densities are oscillating.

Figure 2 shows the real and imaginary parts of eigenvalues of Jacobian matrix $M_{2}$ corresponding to the parameter $Q$. The real parts of all eigenvalues (i.e. $R_{i}<0$, $i=1, \cdots, 4)$ are negative for $Q<Q^{*}(=0.83648)$. At $Q=$ $Q^{*}$ two eigenvalues become purely imaginary (i.e. $R_{1}=$ $R_{2}=0$ and $I_{1}=-I_{2} \neq 0$ ), which confirms that a model system (3) undergoes a Hopf-bifurcation at $Q=0.83648$.

Figure 3 shows the densities of both populations $N$ and $N_{D}$ with respect to the emission rate of external toxicant $Q$. Both densities $N$ and $N_{D}$ become stable at equilibrium level for $Q<Q^{*}$. After crossing the critical value $Q^{*}(=0.83648)$, the equilibrium point losses its stability and a supercritical Hopf-bifurcation occurs (since $\mu_{2}=2.2456 * 10^{-4}>0$ ). Both densities start oscillating around their equilibrium level with stable bifurcating periodic orbits (since $\beta_{2}=-3.5116 * 10^{-7}<0$ ).
Fig. 1 Time-series graph of total and deformed population corresponding to the parameter $Q$
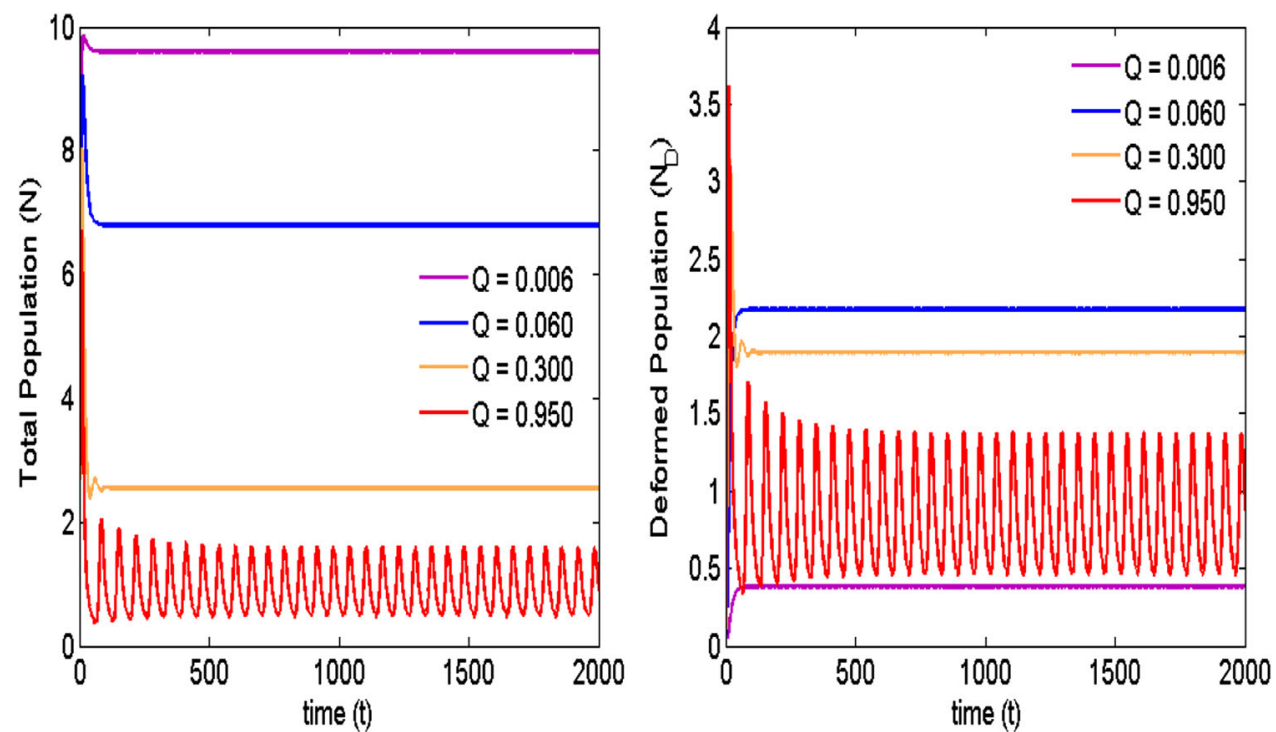
Fig. 2 Real and Imaginary parts of eigenvalues of Jacobian matrix $M_{2}$ respect to the emission rate $Q$
Fig. 3 Dynamic behavior of densities of total and deformed populations for emission rate $Q$
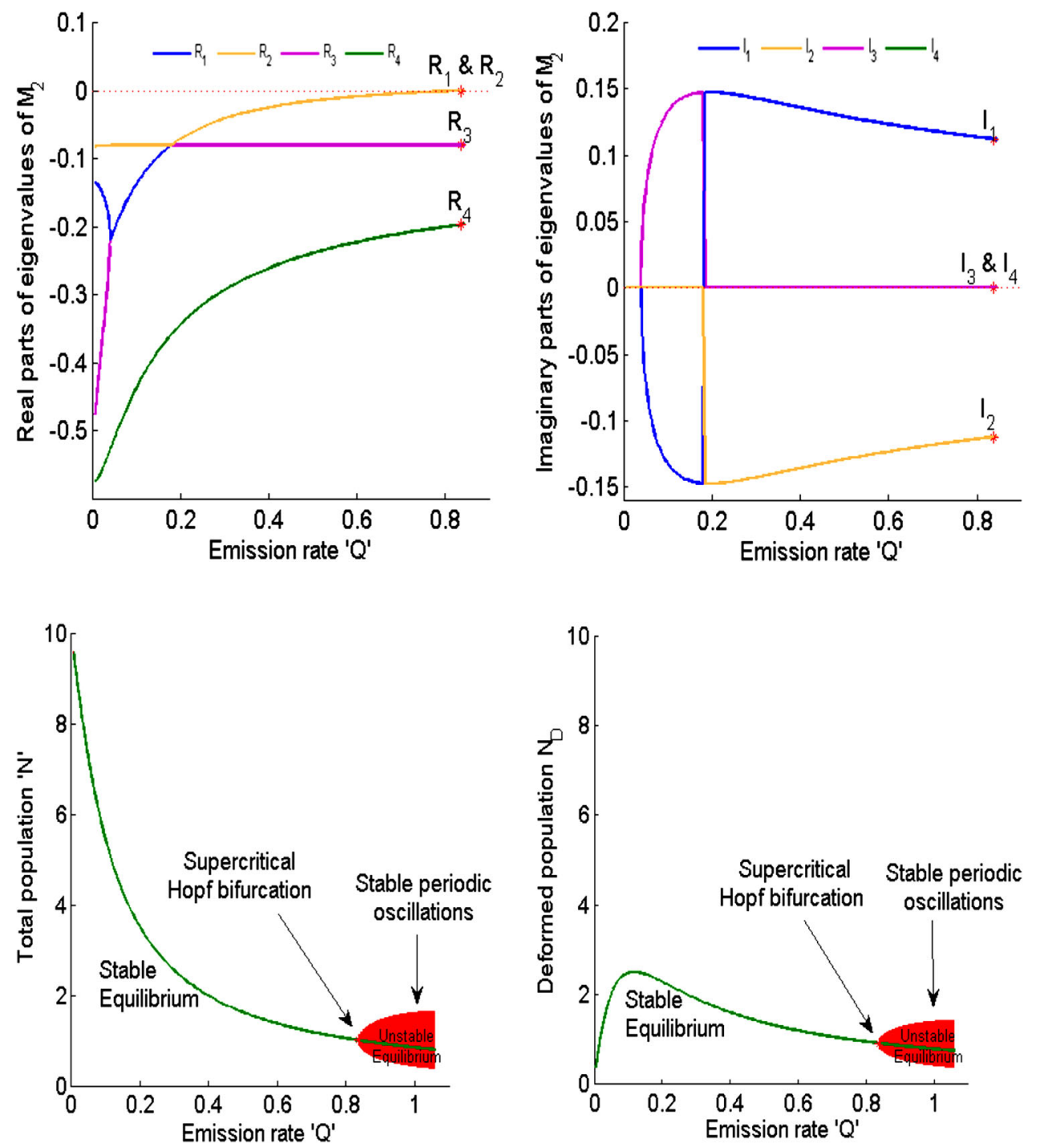

\section{Conclusion}

A mathematical model is proposed to examine the growth of biological species in the case a subclass of species shows deformity, when an external toxicant is constantly emitted in the environment. The analytical results of model show, as emission rate of external toxicant increases, total population density decreases and density of deformed subclass population firstly increase then decrease with total population density. If emission rate crosses the critical value, the model system shows a supercritical Hopf-bifurcation and all the bifurcating periodic solutions of model system are stable.

\section{Appendix A: Proof of the region of attraction $\Omega$}

Proof From the model system (3), we have

$$
\frac{d N}{d t} \leq r N-\frac{r N^{2}}{K_{0}}=r\left(1-\frac{N}{K_{0}}\right) N
$$

Thus, $\lim \sup _{t \rightarrow \infty} N(t) \leq K_{0}$

Also, we have

$$
\begin{aligned}
\frac{d T}{d t}+\frac{d U}{d t} & =Q-\delta T-\beta U-(1-\pi) v N U \\
& \leq Q-\delta_{m}(T+U)
\end{aligned}
$$

where $\delta_{m}=\min (\delta, \beta)$.

Thus, $\lim _{\sup _{t \rightarrow \infty}}(T(t)+U(t)) \leq \frac{Q}{\delta_{m}}$

From the second equation of model (3), we have

$$
\begin{aligned}
\frac{d N_{D}}{d t} & =r_{1} U\left(N-N_{D}\right)-\frac{r N_{D} N}{K(T)}-(\alpha+d) N_{D} \\
& \leq r_{1} \frac{Q}{\delta_{m}}\left(K_{0}-N_{D}\right)-(\alpha+d) N_{D}
\end{aligned}
$$


Thus, $\lim \sup _{t \rightarrow \infty} N_{D}(t) \leq \frac{r_{1} Q K_{0}}{\left[r_{1} Q+\delta_{m}(\alpha+d)\right]}$

providing the region of attraction $\Omega$.

\section{References}

Agrawal AK, Shukla JB (2012) Effect of a toxicant on a biological population causing severe symptoms on a subclass. S Pac J Pure Appl Math 1(1):12-27

Dhooge A, Govaerts W, Kuznetsov YA (2003) MATCONT a MATLAB package for numerical bifurcation analysis of ODEs. ACM Trans Math Softw (TOMS) 29:141-164

Freedman HI, Shukla JB (1991) Models for the effect of toxicant in single species and predator-prey systems. J Math Biol 30: $15-30$

Hassard B, Kazarinoff N, Wan Y (1981) Theory and application of Hopf bifurcation. London mathematical society lecture note series. Cambridge University Press, Cambridge
Jiaqi S, Zhujun J (1995) A new detecting method for conditions of existence of hopf bifurcation. Acta Math Appl Sin 11(1):79-93

Kumar A, Agrawal AK, Hasan A, Misra AK (2016) Modeling the effect of toxicant on the deformity in a subclass of a biological species. Model Earth Syst Environ 2:1-4

Kuznetsov Y (2004) Elements of applied bifurcation theory. Springer, NewYork

Liu W (1994) Criterion of Hopf bifurcation without using eigenvalues. J Math Anal Appl 182:250-256

Seydel R (2009) Practical bifurcation and stability analysis, vol 5, 3rd edn. Springer, Berlin, p 11

Shukla JB, Agrawal AK (1999) Some mathematical models in ecotoxicology; effects of toxicants on biological species. Sadhana 24:25-40

Shukla JB, Agrawal AK, Sinha P, Dubey B (2003) Modeling effects of primary and secondary toxicants on renewable resources. Nat Resour Model 16:99-120 